\title{
La convergence technologique : le cas du véhicule autonome électrique
}

\section{Technological convergence: the case of the autonomous electric vehicle}

\author{
Richard Le Goff ${ }^{1}$ \\ ${ }^{1}$ Unité d'Economie Appliquée, ENSTA Paris, i3-CRG Ecole Polytechnique - CNRS (UMR 9217), Institut Polytechnique de \\ Paris, France, richard.le-goff@ensta-paris.fr
}

\begin{abstract}
RÉSUMÉ. L'émergence industrielle fondée sur des mouvements de fusion totale ou partielle de secteurs anciennement organisés en silos reste encore aujourd'hui un angle mort de l'économie industrielle. L'approche des " marchés augmentés " constitue une alternative aux approches de cycles de vie industriels et de systèmes d'innovation technologique pour apporter des éléments d'interprétation de mouvements stratégiques qui caractérisent des dynamiques sectorielles significatives actuellement. Nous proposons dans l'article d'associer le technologique et le sectoriel en utilisant la théorie de la dominance économique (TDE). La TDE permet de vérifier, en amont de toute tentative de validation empirique au niveau " production " et " marchés ", que les fondements technologiques de l'émergence sectorielle par la convergence sont bien présents.

ABSTRACT. Industrial emergence based on total or partial mergers of sectors formerly organized in silos remains a blind spot in industrial economics. The "augmented markets" approach constitutes an alternative to the industrial life cycle and the technological innovation system approaches to provide elements for interpreting strategic movements that characterize significant sectoral dynamics today. In this paper, we propose using the economic dominance theory (EDT) to associate the technology and the sector. The EDT ensures, upstream of any attempt of empirical validation at the "production" and "market" levels, that the technological foundations of sectoral emergence through convergence are indeed present.

MOTS-CLÉS. Marchés augmentés, véhicule autonome électrique, théorie de la dominance économique.

KEYWORDS. augmented markets, autonomous electric vehicle, economic dominance theory.
\end{abstract}

\section{Introduction}

Les outils de la théorie mathématique des graphes pour étudier les transformations de systèmes d'innovation dans le temps à partir de données de brevets sont le plus souvent associés à des structures de cooccurrences, où les combinaisons technologiques au sein des brevets et les déposants de ces brevets s'entremêlent pour laisser apparaître des relations secteurs / technologies mouvantes (Fauconnet, ce numéro). Dans ce cadre, la technologie apparaît comme une force de convergence ou d'éclatement sectoriel extrêmement puissante, à la manière dont l'anticipent [BER 08] et [BER 15]. Il est cependant difficile à partir de ces outils de percevoir le point final de l'évolution de ces relations, i.e. l'émergence de nouveaux secteurs productifs stables et de nouveaux marchés.

Pour envisager ce terme, ce sont les approches de cycles de vie industriels qui sont le plus souvent appelées [KLE 96]. Le cycle de vie d'une industrie est caractérisé par un certain nombre de régularités statistiques qui se rapportent à la démographie des firmes et à l'intensité relative des innovations de produits (forte dans les premiers moments du cycle) et de procédés (qui dominent ensuite). Cette dynamique aboutit à une situation de concurrence oligopolistique stable en phase de maturité à la suite d'un shake out et - caractéristique essentielle - les firmes en place en cette période de maturité sont présentes dès les premiers instants de la vie de l'industrie. L'émergence industrielle résulte rarement, dans cette perspective, de rapprochement d'industries bien installées qui verraient leurs modes d'innovation et de production bouleversés par un élément exogène ou endogène déclencheur. Et les cycles industriels semblent de plus prendre des formes nouvelles au cours des dernières décennies. Comme le montrent [GRE 06] qui étudient les cycles de vie d'industries high tech depuis les années 1970, de nouvelles entreprises peuvent rester viables après la phase de décollage, ce qui fait que 
l'industrie mature est composée d' «anciennes» comme de «nouvelles» firmes, l'avantage des premières en termes de procédés étant compensé par l'avantage des dernières en termes de produits. L'organisation en réseau, qui permet de gérer les asymétries de connaissances et de créer des synergies autour d'elles, se développe au point de devenir un mode normal d'organisation dans ces industries high techs.

Dans cet article, nous raisonnons différemment de l'approche en termes de cycle de vie industriel, en adoptant un point de vue plus téléologique : nous posons l'émergence d'une nouvelle industrie, celle des véhicules automobiles autonomes électriques (VAE). Ces dernières années, nous observons l'apparition de nouveaux produits : des véhicules à propulsion électrique et à conduite autonome (aptes à rouler sans intervention d'un conducteur), qui apparaissent comme une véritable innovation de rupture, technologique et commerciale comme sociétale ([LAM 16], [MEY 17]).

Ces nouveaux produits sont le résultat d'initiatives d'institutions de recherche et d'entreprises des secteurs des transports, des télécommunications et de l'énergie. La dynamique contemporaine de décloisonnement entre ces industries, auparavant organisées en silos, modifie les frontières des marchés. Les entreprises de ces industries cherchent à exploiter les mêmes bases intangibles (connaissances, informations, etc.) ou physiques (infrastructures, technologies, etc.) à différents niveaux de la chaîne de valeur [BAI 16]. Elles modifient potentiellement les frontières entre ces trois industries et créent de nouvelles synergies dans le domaine de la recherche et du développement (R\&D). Nous cherchons à savoir si la convergence entre les marchés du transport, des télécommunications et de l'énergie que traduit l'émergence du VAE est précédée d'une convergence technologique. Est-ce que les manières d'inventer se sont transformées dans le temps de façon à faire apparaître des synergies entre des domaines technologiques de l'électronique-informatiquetélécommunications, de l'énergie et des transports au cœur de la nouvelle industrie ? Nous proposons ici une méthode et des métriques spécifiques pour répondre à cette question. Nous tentons en effet de repérer les signes technologiques de l'émergence du VAE en utilisant les outils structuraux de la théorie de la dominance économique (TDE).

\section{La théorie de la dominance économique appliquée à l'étude des flux de connaissances technologiques}

La théorie de la dominance économique (TDE) est depuis récemment utilisée pour étudier des matrices de flux technologiques construites à partir de données de brevets [LEB 17]. Nous utilisons la base de données PATSTAT (version automne 2014) pour repérer les brevets, les technologies auxquelles ils s'associent (nomenclature de l'office mondial des brevets - WIPO - à 4 digits, le niveau des sous-classes technologiques) et leurs citations (donc les technologies issues de ces citations). Une matrice de flux technologiques est une matrice carrée ou chaque ligne et chaque colonne correspond à une entrée de la nomenclature WIPO. Les technologies citées sont en ligne, les technologies citant sont en colonne.

Imaginons qu'un brevet se réfère aux technologies $\mathrm{A}$ et $\mathrm{B}$ et qu'il cite un brevet se référant aux technologies A et C. Dans ce cas, les flux de connaissances technologiques vont de A et C vers A et B, et nous faisons en sorte que la somme des flux pour cette citation vaille 1 précisément, de sorte que la somme des arguments de la matrice des flux soit strictement égale au nombre de citations. Dans l'exemple, le lien allant de $\mathrm{A}$ à $\mathrm{A}$ (autocitation) vaut 0,25 , de même que celui allant de $\mathrm{A}$ à $\mathrm{C}$, de $\mathrm{C}$ à $\mathrm{A}$ et de $\mathrm{C}$ à $\mathrm{B}$. Au total, la matrice de flux technologiques nous informe sur la manière dont, à une date donnée, on combine des technologies différentes qui ont été inventées dans le passé pour produire de l'invention à cette date. Ce qui nous intéresse, dans cet article, c'est la façon dont ces manières d'inventer se sont transformées au fil du temps.

Grâce à PATSTAT, nous construisons des matrices de flux technologiques pour les années allant de 1980 à 2010 avec un incrément de 5 ans, soit 7 périodes en tout. Les brevets retenus sont demandés à 
l'Office Européen des Brevets (OEB), à l'office américain (USPTO), à l'office japonais (JPO) ou dans les offices nationaux des pays de l'Union Européenne à 15 membres (Europe occidentale). Une même invention pouvant être déposée dans plusieurs offices, c'est au niveau des «familles » de brevets que nous identifions les citations et les technologies, c'est-à-dire des regroupements de brevets pour des inventions identiques déposées dans des offices différents.

Notre base de données est donc constituée de citations au niveau des familles et des technologies associées à chacune des familles appelées dans les citations. Nous obtenons les effectifs reportés dans le tableau 1.

\begin{tabular}{|c|c|c|}
\hline Année & \# citations & \# technologies \\
\hline 1980 & 1111746 & 1489565 \\
\hline 1985 & 1135186 & 1741710 \\
\hline 1990 & 1414733 & 2039863 \\
\hline 1995 & 1789974 & 2270692 \\
\hline 2000 & 3218835 & 3709339 \\
\hline 2005 & 4310374 & 5680044 \\
\hline 2010 & 3138132 & 4919580 \\
\hline
\end{tabular}

Tableau 1. Effectifs

Il y a 643 technologies uniques (codes WIPO 4 digits) qui sont appelées dans cette masse, ce qui fait que les matrices de flux technologiques sont de format $643 \times 643$. Chacune de ces technologies fait partie d'un domaine et d'un sous-domaine technologique définis par l'Observatoire des Sciences et Techniques ([OST 10] ; tableau 2). C'est au niveau des sous-domaines que nous essayons d'identifier la convergence technologique dans le véhicule autonome électrique (VAE). Ces sous-domaines sont surlignés en gris dans le tableau 2.

Nous nous intéressons aux relations entre sous-domaines technologiques et aux transformations de ces relations dans le temps. Nous neutralisons les autocitations en posant que la relation entre une même technologie est nulle. L'analyse se concentre sur les liens entre des technologies différentes.

\begin{tabular}{|c|c|c|c|}
\hline Domaines & Sous-domaines & Domaines & Sous-domaines \\
\hline \multirow[t]{5}{*}{ Électronique-électricité } & Composants électriques & \multirow[t]{3}{*}{ Pharmacie-biotechnologies } & \multirow{3}{*}{$\begin{array}{l}\text { Biotechnologies } \\
\text { Pharmacie-cosmétiques } \\
\text { Produits agricoles et alimentaires }\end{array}$} \\
\hline & \multirow{3}{*}{$\begin{array}{l}\text { Audiovisuel } \\
\text { Télécommunications } \\
\text { Informatique }\end{array}$} & & \\
\hline & & & \\
\hline & & \multirow[t]{5}{*}{ Procédés industriels } & Procédés techniques \\
\hline & Semi-conducteurs & & Manutention-imprimerie \\
\hline \multirow[t]{4}{*}{ Instrumentation } & \multirow{4}{*}{$\begin{array}{l}\text { Optique } \\
\text { Analyse-mesure-contrôle } \\
\text { Ingénierie médicale } \\
\text { Techniques nucléaires }\end{array}$} & & Travail matériaux \\
\hline & & & Environnement-pollution \\
\hline & & & Appareils agricoles et alimentation \\
\hline & & \multirow[t]{6}{*}{ Machines-mécanique-transports } & Machines-outils \\
\hline \multirow[t]{5}{*}{ Chimie-matériaux } & \multirow{5}{*}{$\begin{array}{l}\text { Chimie organique } \\
\text { Chimie macromoléculaire } \\
\text { Chimie de base } \\
\text { Traitements surface } \\
\text { Matériaux-métallurgie }\end{array}$} & & Moteurs-pompes-turbines \\
\hline & & & Procédés thermiques \\
\hline & & & Composants mécaniques \\
\hline & & & Transports \\
\hline & & & Spatial-armement \\
\hline & & Consommation des ménages-BTP & $\begin{array}{l}\text { Consommation des ménages } \\
\text { BTP }\end{array}$ \\
\hline
\end{tabular}

Tableau 2. Nomenclature technologique [OST 10] 
La théorie de la dominance économique (TDE) va nous permettre de représenter la forme des liens entre technologies, au niveau de la structure dans son ensemble ou au niveau de «parties » de cette structure. La TDE va pouvoir produire une mesure de l'importance relative des relations de «dépendance » au sein de la structure et de ses parties, i.e. l'importance des relations arborescentes ou hiérarchiques - entre les technologies, et une mesure de l'importance relative des « interdépendances » au sein de la structure et de ses parties, i.e. des relations circulaires, génératrices de feedbacks et d'effets d'amplification (voir Lebert, ce numéro).

L'analyse des convergences technologiques dans le cadre de la TDE est donc liée à la décomposition de la structure globale en «parties » (i.e. en groupes de pôles), aux caractéristiques de ces parties et aux relations qu'elles entretiennent entre elles. La valeur référence de l'analyse structurale - le déterminant matriciel [LAN 13] - est alors appréhendé d'une manière particulière : le déterminant de la structure des échanges est inférieur ou égal au produit des déterminants des parties (théorème de la partition; cf. figure 1). La différence entre le produit des déterminants des parties et $D$ (valeur du déterminant) mesure l'interdépendance entre ces parties (le poids des circularités). Dans un cas extrême où chaque pôle constitue une partie, la différence entre le produit des déterminants de ces parties (le produit des termes diagonaux de la structure des échanges, produit nécessairement égal à 1 s'il n'y a pas d'autoconsommation) et le déterminant de la structure entière mesure l'interdépendance générale entre les pôles de la structure. Dans l'autre cas extrême où l'ensemble des pôles est inclus dans une grande partie unique, la différence (nulle par construction) indique que l'ensemble des relations d'interdépendance au sein de la structure est internalisé par la partie.

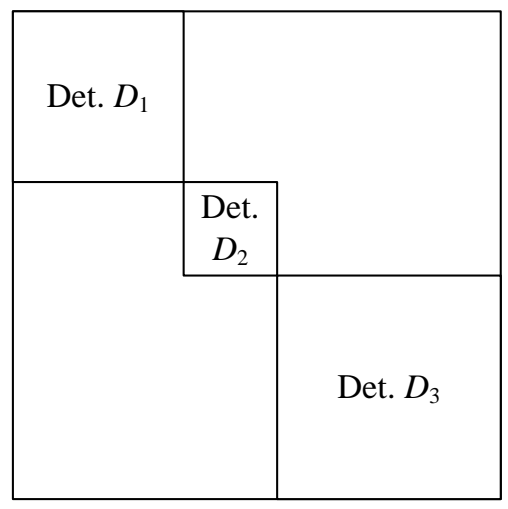

La valeur $\boldsymbol{D}$ du déterminant est plus petite que le produit $\boldsymbol{D}_{1} \times \boldsymbol{D}_{2} \times \boldsymbol{D}_{3}$ sauf s'il n'existe aucun circuit liant les pôles de deux ou trois sousmatrices définies par la partition. Ainsi, l'interdépendance entre les parties est égale à $\Gamma_{1-2-3}=D_{1} \times D_{2} \times D_{3}-D$

Figure 1. Illustration du théorème de la partition [LAN 13]

Dans le cas de l'article, nous identifions 7 parties, dont 6 constituent le système « véhicule autonome et électrique » (tableau 2), la dernière regroupant le reste de la structure. Le tableau 3 recense les technologies (nomenclature WIPO 4 digits) faisant partie des 6 sous-domaines du VAE. Dans un premier temps intéressons-nous aux caractéristiques structurales des 6 parties combinées entre elles (soit 149 technologies sur les 643 que comprend la structure) comparées à celle de la structure globale.

La sous-structure «véhicule autonome et électrique »(VAE) est un peu plus dense que la structure globale (la densité correspondant au rapport entre le nombre de connexions entre les composantes technologiques de la sous-structure rapporté au nombre de connexions possibles entre ces composantes). Cette densité a tendance à croître avec le temps (+ 0,2 pt entre 1980 et 2005), mais cette tendance ne se différencie pas trop de celle qui caractérise la structure dans son ensemble (figure 2). Par contre, l'intensité moyenne des connexions inter-technologiques au sein de la partie est beaucoup plus élevée (figure 3), et l'écart s'accentue avec le temps (valeur sommée des flux dans la partie divisée par le nombre de connexions inter-technologiques existantes). Nous avons là un premier indice de l'imbrication croissante des technologies constituant le VAE au cours des dernières décennies. 
F21H, F21K, F21L, F21S, F21V, F21W, F21Y, H01B, H01C, H01F, H01G, H01H, 29 H01J, H01K, H01M, H01R, H02B, H02G, H02H, H02J, H02K, H02M, H02N, H02P, H02S, H05B, H05C, H05F, H05K

Télécommunications H01P, H03B, H03C, H03D, H03H, H03K, H03L, H04B, H04H, H04J, H04K, H04L, H04M, H04Q, H04W

\section{Informatique}

Moteurs-pompes-turbines

G06C, G06D, G06E, G06F, G06G, G06J, G06K, G06M, G06N, G06Q, G06T, G10L

F01B, F01C, F01D, F01K, F01L, F01M, F01P, F02B, F02C, F02D, F02F, F02G, 26 F02K, F02M, F02N, F02P, F03B, F03C, F03D, F03G, F03H, F04B, F04C, F04D, F04F, F23R

Procédés thermiques F22B, F22D, F22G, F23B, F23C, F23D, F23H, F23K, F23L, F23M, F23N, F24B, 25 F24C, F24D, F24F, F24H, F24J, F25B, F27B, F27D, F28B, F28C, F28D, F28F, F28G

\section{Transports}

B60B, B60C, B60D, B60F, B60G, B60H, B60J, B60K, B60L, B60M, B60N, B60P, 42 B60Q, B60R, B60S, B60T, B60V, B60W, B61B, B61C, B61D, B61F, B61G, B61H, $\mathrm{B} 61 \mathrm{~J}, \mathrm{~B} 61 \mathrm{~K}, \mathrm{~B} 61 \mathrm{~L}, \mathrm{~B} 62 \mathrm{~B}, \mathrm{~B} 62 \mathrm{C}, \mathrm{B} 62 \mathrm{D}, \mathrm{B} 62 \mathrm{H}, \mathrm{B} 62 \mathrm{~J}, \mathrm{~B} 62 \mathrm{~K}, \mathrm{~B} 62 \mathrm{~L}, \mathrm{~B} 62 \mathrm{M}, \mathrm{B} 63 \mathrm{~B}$, $\mathrm{B} 63 \mathrm{C}, \mathrm{B} 63 \mathrm{H}, \mathrm{B} 64 \mathrm{~B}, \mathrm{~B} 64 \mathrm{C}, \mathrm{B} 64 \mathrm{D}, \mathrm{B} 64 \mathrm{~F}$

Tableau 3. Composition technologique du $V A E^{1}$

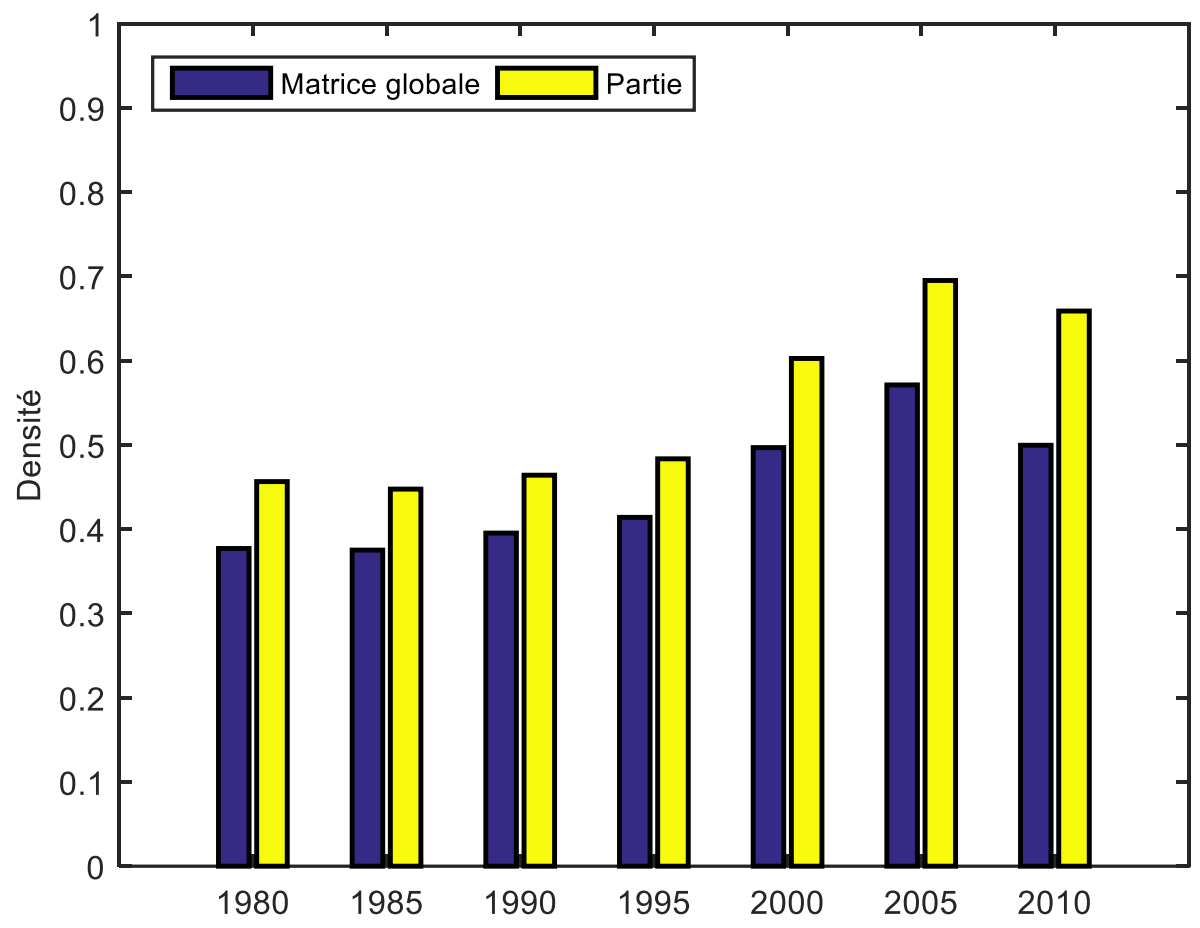

Figure 2. Densité de la partie VAE et de la structure globale

\footnotetext{
${ }^{1}$ https://www.wipo.int/classifications/ipc/fr/ 


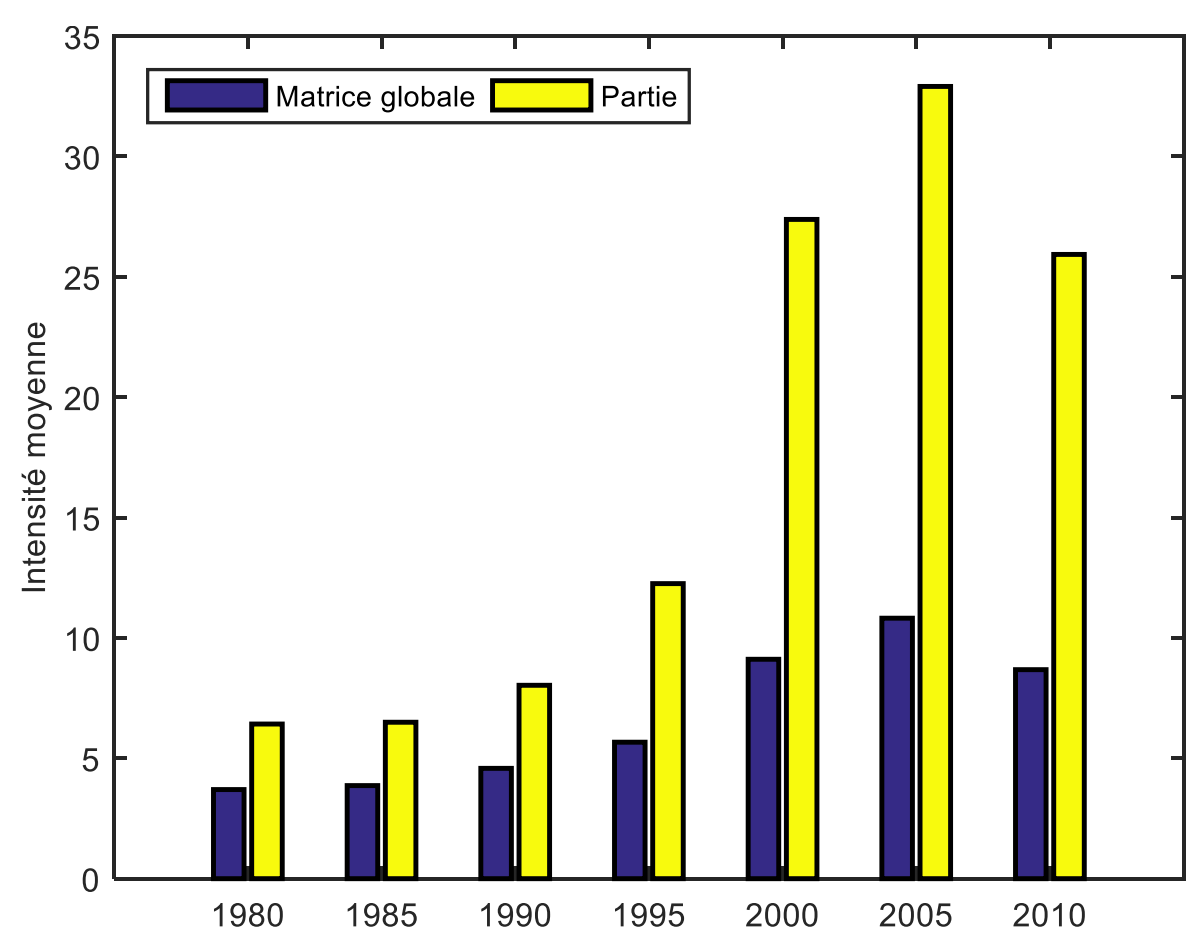

Figure 3. Intensité moyenne des connexions dans le VAE et la structure globale

\section{Principaux résultats et discussion}

Comment les synergies technologiques se sont recomposées dans les processus de production des inventions durant la période 1980-2010 ? Nous allons mesurer des proximités entre l'ensemble des parties de la structure des échanges technologiques prises deux à deux (les 30 sous-domaines technologiques identifiés dans le tableau 2) afin de quantifier ces changements structurels. Pour cela, nous utilisons le théorème de la partition présenté dans la section précédente. Considérons 3 parties, les parties $p_{1}$ et $p_{2}$ contenant les ensembles technologiques attachés aux sous-domaines dont nous souhaitons mesurer la proximité, la partie $P$ correspondant à la sous-structure fusionnant ces deux ensembles. Nous définissons la proximité $d_{P}$ comme l'interdépendance relative portée par la partition de $P$.

$$
d_{P}=\frac{D_{p_{1}} D_{p_{2}}}{D_{P}}
$$

L'indice $d_{P}$ est égal au rapport entre l'interdépendance de la partition de $P$ et la dépendance présente dans $P$. Plus $d_{P}$ est élevé, plus la partition de $P$ révèle de « synergies » (poids des circularités) entre $p_{1}$ et $p_{2}$. Nous obtenons une matrice symétrique de proximité de format $30 \times 30$. Nous procédons à une normalisation afin que la valeur de 1 pour $d_{P}$ quel que soit $P$ indique la proximité inter-technologique la plus forte, la valeur de 0 la proximité la plus faible. Cette matrice de proximité normalisée est utilisée pour produire un dendrogramme pour chaque année de l'étude.

Les figures 4 et 5 reproduisent les dendrogrammes de proximités inter-technologiques pour les années 1980 et 2010. Par exemple, les sous-domaines «Transports » et «Composants mécaniques » sont fortement synergétiques en 1980 et constituent à eux deux un cluster technologique éloigné du reste de la structure. En 2010, ce cluster s'associe plus étroitement au trio «BTP / Consommation des ménages / Ingénierie médicale » qui était déjà synergétique trente ans plus tôt. 


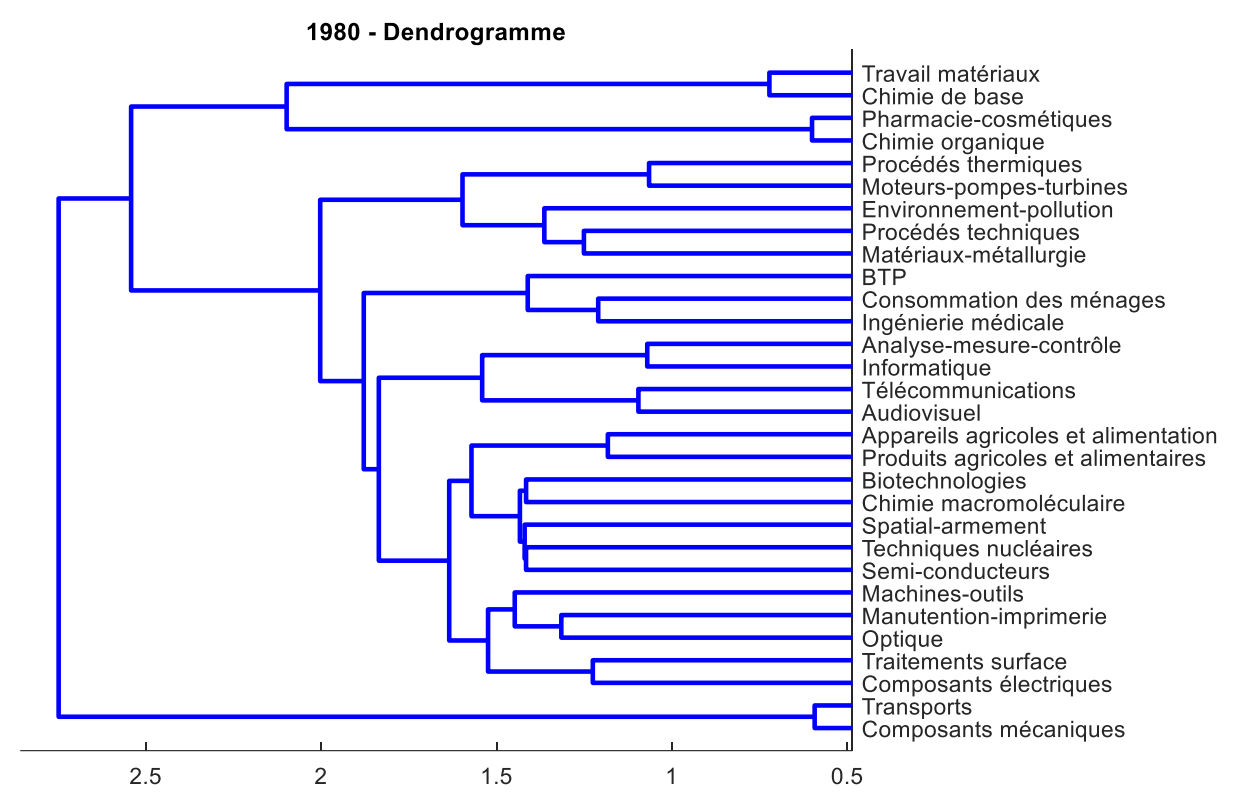

Figure 4. Proximités inter-technologiques pour l'année 1980

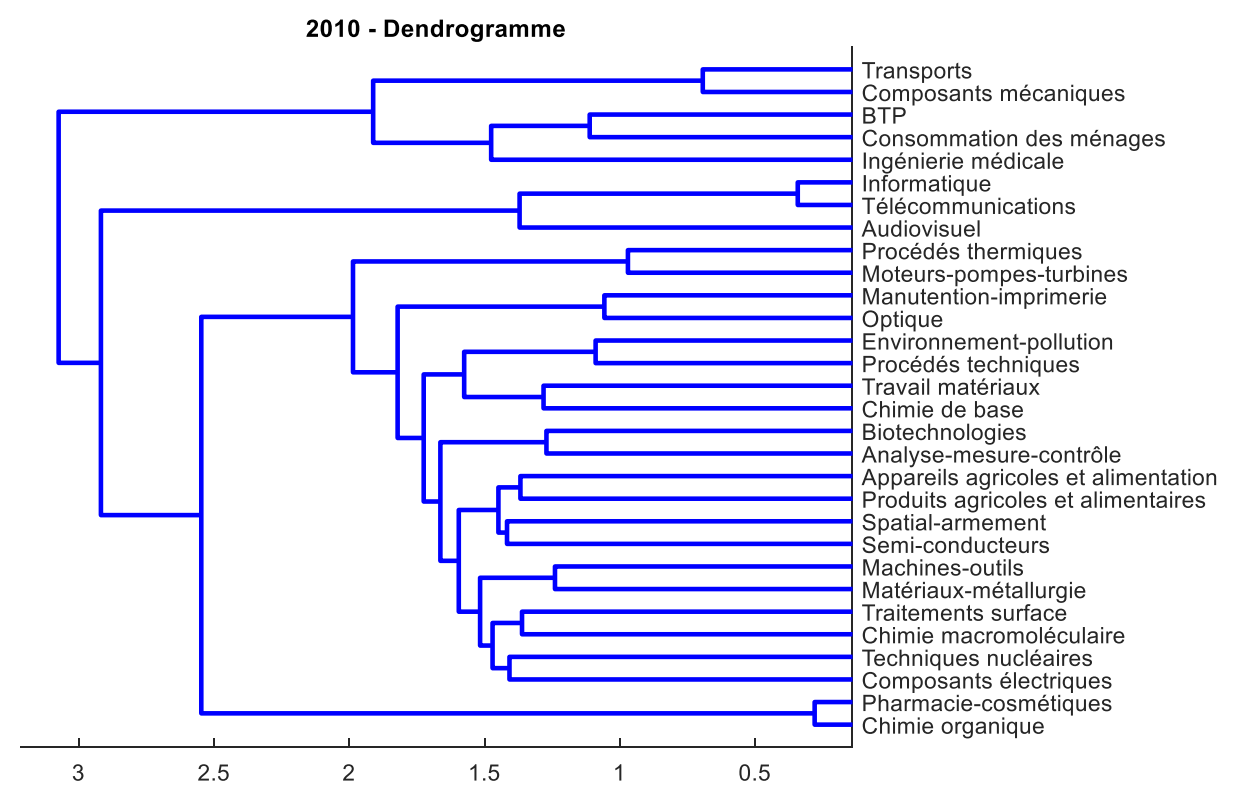

Figure 5. Proximités inter-technologiques pour l'année 2010

Cette lecture descriptive des transformations structurelles entre 1980 et 2010 peut être étayée par un indice de corrélation de Pearson appliqué aux matrices de proximités prises deux à deux (figure 6).

Les corrélations sur les valeurs des proximités inter-technologiques restent fortes sur toute la période (scores supérieurs à 0,8) et l'année 2000 apparaît celle qui est la plus dissemblable des autres. Globalement, les manières d'inventer changent peu durant la période. Les synergies que l'on trouvait il y a 30 ans sont pour la plupart présentes aujourd'hui.

Qu'en est-il pour le système VAE ? Nous appliquons l'indice $d_{P}$ à la sous-structure $P$ additionnant les 149 technologies des 6 sous-domaines qui le constituent (figure 7). 


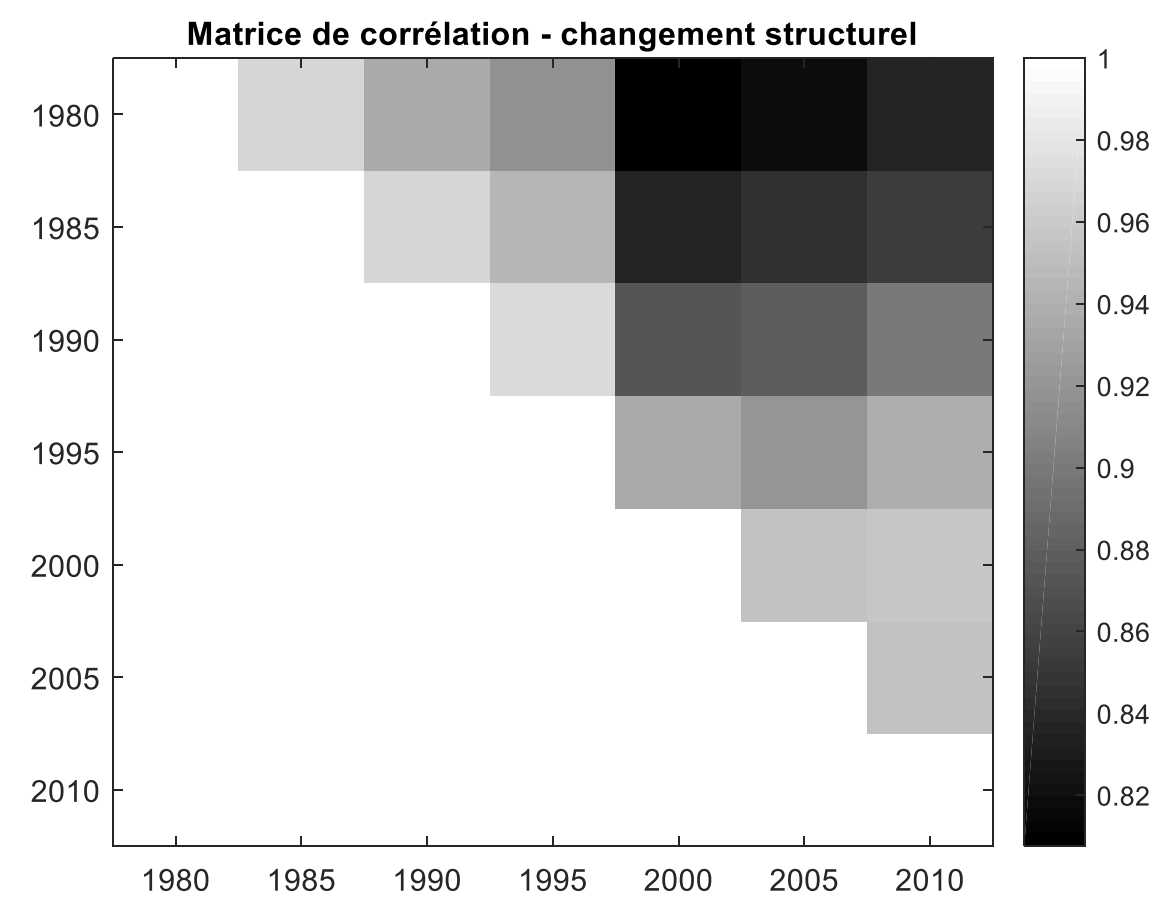

Figure 6. Corrélations de Pearson sur les proximités inter-technologiques

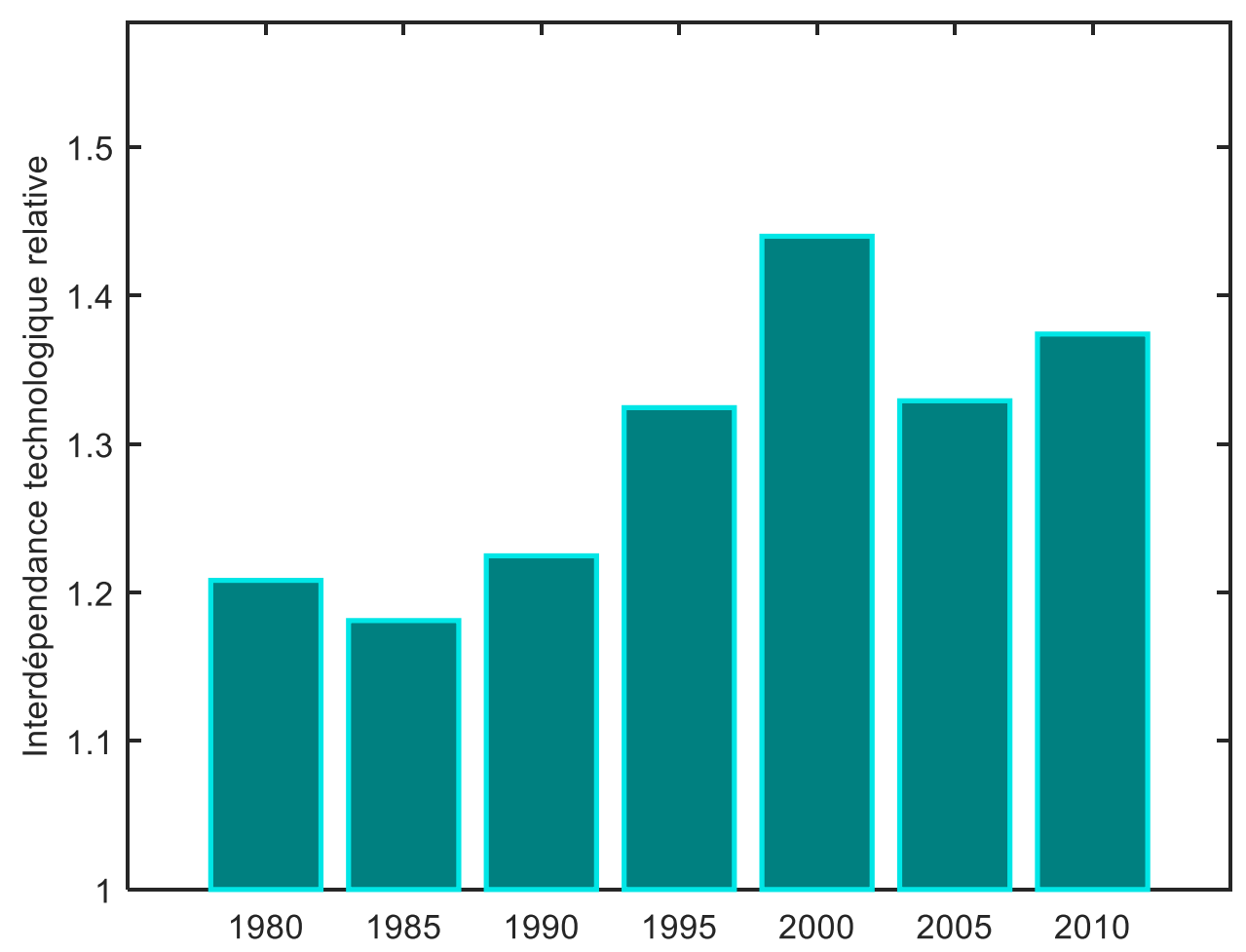

Figure 7. Convergence technologique

L'intensité des synergies est multipliée par deux environ sur la période $(+0,2 \mathrm{pt})$. Les scores obtenus pour $d_{P}$ sont très élevés lorsqu'on les compare à des partitions aléatoires de la structure. La figure 8 reporte les z-scores standardisés de la combinaison des sous-domaines technologiques correspondant au système VAE (relativement à l'ensemble des 593000 combinaisons de 6 sous-domaines dans les 30 sous-domaines identifiés par [OST 10]). Ces z-scores sont très supérieurs à 1 et ont tendance à croître avec le temps, pour aboutir à un maximum d'environ 4,5 pour l'année 2010. Autrement dit, 
l'association Composants électriques, Télécommunications, Informatique, Moteurs-pompes-turbines, Procédés thermiques et Transports produit des synergies de plus en plus remarquables dans le paysage technologique global, ce qui vient confirmer l'imbrication croissante des technologies constituant le système VAE au cours des dernières décennies.

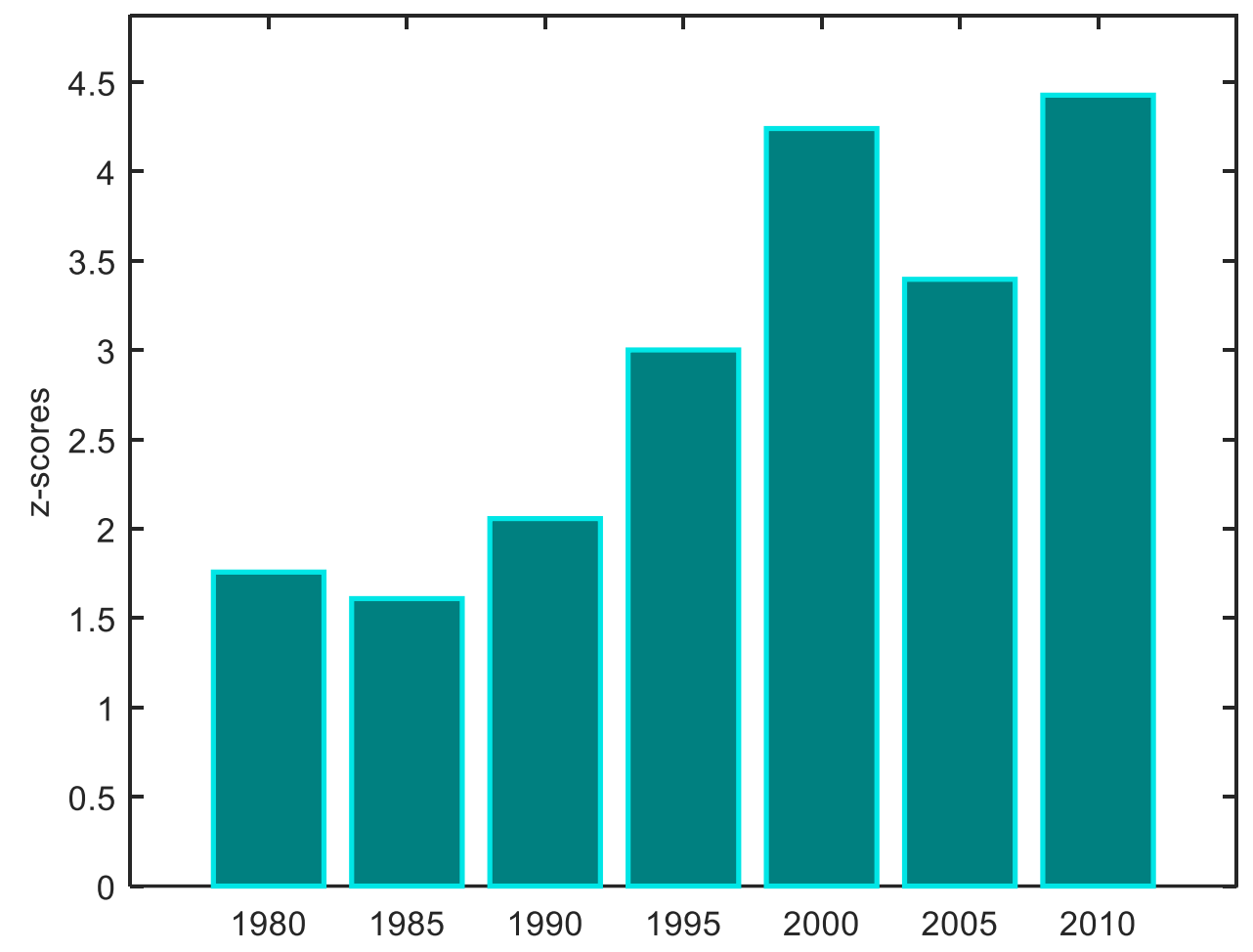

Figure 8. z-scores standardisés pour le système VAE

Certaines industries semblent aujourd'hui adopter une logique de décloisonnement. Cette logique conduit à abandonner l'organisation en compartiments en faveur d'une organisation systémique qui capitalise non seulement sur le regroupement des activités, mais aussi sur leur étroite interconnexion. A la suite de [BAI 16], nous pensons que les secteurs de l'énergie, des transports et du numérique sont ceux qui reflètent le mieux ce changement de paradigme. Par exemple, Google, en tant qu'infomédiaire, a élargi le champ de ses activités avant de modifier sa structure d'entreprise (création de la maison mère Alphabet Inc. en 2015) en pénétrant les domaines de l'énergie (Google Energy, créé en 2009) et des transports (le projet de véhicule autonome Google Car au sein du X Lab) dans le cadre d'une stratégie de diversification offensive visant à exploiter des synergies fonctionnelles, productives et commerciales. Nous soutenons que Google développe l'archétype d'une «stratégie de marché augmentée ». Cette stratégie peut être définie comme l'extension, ou la création, d'un marché par l'établissement de réseaux reliant deux ou plusieurs marchés autonomes (différenciés par leur structure et leurs acteurs). Elle est rendue possible par le décloisonnement de secteurs qui partagent désormais occasionnellement ou non - les mêmes fondements physiques (infrastructures, technologie, etc.) et / ou immatériels (connaissances, informations, etc.), à différents niveaux de leurs chaînes de valeur respectives. Une telle stratégie capitalise sur le décloisonnement sectoriel, notamment par la mutualisation des infrastructures de services dans les domaines de l'économie numérique, de l'énergie et des transports. En outre, les «marchés augmentés » appuient l'émergence d'innovations de produits que l'on peut appeler «produits augmentés». Le VAE en fait partie. En amont, la «technologie augmentée », celle dont nous cherchons à montrer l'existence ici, participe à l'extension des fonctionnalités du produit [LAN 66] par rapport à celles qui sont habituellement proposées sur un segment de marché donné. 
Notre article ne prouve certainement pas que le VAE résulte d'une stratégie délibérée visant à « accroître le marché » en créant un socle d'intangibles constitué de brevets issus d'un effort de R\&D parfaitement piloté et mené par des décisions stratégiques prises par les conseils d'administration d'entreprises pionnières. Toutefois, il contribue à appuyer l'hypothèse d'une origine technologique des innovations de produits VAE fondées sur l'exploitation de synergies croissantes entre domaines de compétences énergie / transports / informatique-électronique-télécommunications. Par conséquent, il semble que les stratégies de développement des entreprises installées appartenant aux trois secteurs d'origine pourraient se concevoir et s'évaluer dorénavant à l'aune du décloisonnement sectoriel illustré par le VAE.

\section{Conclusion}

L'émergence industrielle fondée sur des mouvements de fusion totale ou partielle de secteurs anciennement organisés en silos reste encore aujourd'hui un angle mort de l'économie industrielle. Les approches en termes de cycle de vie des industries ne l'ont pas formalisée, celles en termes de systèmes d'innovation mêlant le technologique et le sectoriel n'ont, à ce jour, pas encore produits de " preuves de concept » satisfaisantes selon nous. L'approche des « marchés augmentés » constitue une alternative pour apporter des éléments d'interprétation de mouvements stratégiques qui, somme toute, caractérisent des dynamiques industrielles significatives actuellement. Là aussi, le cadre d'analyse est à compléter. Nous proposons dans l'article d'associer le technologique et le sectoriel en utilisant la théorie de la dominance économique. La TDE permet de vérifier, en amont de toute tentative de validation empirique au niveau «production » et «marchés », que les fondements technologiques de l'émergence sectorielle par la convergence sont bien présents. Dans cet article, cela signifie que les synergies technologiques se construisent historiquement avant les synergies productives et commerciales. Les premières permettent d'anticiper les secondes. A la manière dont il est construit, cet enchaînement historique n'est cependant pas suffisant. Il est d'une part linéaire, gommant les effets de rétroaction possibles du marché augmenté sur la technologie augmentée. Elle ne construit pas non plus de liens clairs entre la technologie et le produit (parmi l'ensemble des inventions synergétiques dans les six domaines étudiés, lesquelles contribuent effectivement au développement de nouveaux produits ou procédés augmentés ?), et elle omet encore les relations amont avec la science. Ces trois limites constituent autant de programmes de recherche pour faire de l'approche des marchés augmentés une théorie complète de l'émergence industrielle par décloisonnement.

\section{Bibliographie}

[BAI 16] BAINEE J., LE GOFF R., «Le marché augmenté de l'énergie, des transports et du numérique », Innovations, ${ }^{\circ}$ 50, p. 95-118, 2016.

[BER 08] BergeK A., Jacobsson S., CARLSSON B., LindMARK S., Rickne A., « Analyzing the functional dynamics of technological innovation systems: a scheme of analysis », Research Policy, vol. 37, p. 407-429, 2008.

[BER 15] Bergek A., HekKert M., Jacobsson S., Markard J., SAndén B., TrufFer B., « Technological innovation systems in contexts: conceptualizing contextual structures and interaction dynamics », Environmental Innovation and Societal Transitions, vol. 16, p. 51-64, 2015.

[GRE 06] Grebel T., Krafft J., SAViotti P.P., « On the life cycle of knowledge intensive sectors », Revue de l'OFCE, $\mathrm{n}^{\circ}$ spécial, juin, p. 63-85, 2006.

[KLE 96] KLEPPER S., «Entry, exit, growth, and innovation over the product life cycle », American Economic Review, vol. 86, p. 562-583, 1996.

[LAM 16] LAmotte R., DE PAlma A., Geroliminis N., "Sharing the road: the economics of autonomous vehicles », hal-01281425, 2016.

[LAN 66] LANCASTER K.J., «A new approach to consumer theory », Journal of Political Economy, vol. 54, p. 132-157, 1966. 
[LAN 13] LANTNER R., LEBERT D., «Dominance, dependence and interdependence in linear structures: a theoretical model and an application to the international trade flows », CES Working Papers, ${ }^{\circ}{ }^{13043,} 2013$.

[LEB 17] LEBERT D., El Younsi H., Dynamiques des spécialisations internationales, ISTE Press, Londres, 2017.

[MEY 17] MEYeR J., BECKeR H., BÖSCH P.M., AXHAUSEN K.W., «Autonomous vehicles: The next jump in accessibilities? », Research in Transportation Economics, vol. 62, p. 80-91, 2017.

[OST 10] OST, Indicateurs de sciences et de technologies, Rapport biennal de l'Observatoire des Sciences et des Technologies, Paris, 2010. 